ఠ

\title{
Expression and clinical significance of the long non-coding RNA PVTI in human gastric cancer
}

This article was published in the following Dove Press journal:

OncoTargets and Therapy

18 September 2014

Number of times this article has been viewed

\section{Jian Dingl,* \\ Dan $\mathrm{Li}^{2}, *$ \\ Minzhen Gong' \\ Jinpo Wang' \\ Xunru Huang' \\ Ting $\mathrm{Wu}^{\prime}$ \\ Chengdang Wang'}

'Digestive Department of the First Affiliated Hospital of Fujian Medical University, Fuzhou, Fujian, People's Republic of China; ${ }^{2}$ Digestive

Department of Union Hospital of Fujian Medical University, Fuzhou, Fujian, People's Republic of China

*These authors contributed equally to this work
Correspondence: Jian Ding Digestive Department of the First Affiliated Hospital of Fujian Medical University, Fuzhou, Fujian,

People's Republic of China

Email docdingjian@।63.com
Background: Highly sensitive markers are urgently needed for the diagnosis and grading of gastric cancer and for managing drug resistance. The recent identification of long-non-coding RNAs (lncRNAs) has provided new approaches for resolving this challenge. The aim of this study was to screen and identify new biomarkers for human gastric cancer from lncRNAs.

Methods: First, we used lncRNA microarrays to conduct a preliminary screening for candidate lncRNAs of gastric cancer biomarkers in both human gastric cancer tissues and in two gastric cancer cell lines, SGC7901 cells and paclitaxel-resistant SGC7901 cells. The lncRNA plasmacytoma variant translocation 1 ( $P V T 1)$ was found to exhibit higher expression in both gastric cancer tissues and the SGC7901 paclitaxel-resistant cell line. Quantitative polymerase chain reaction was used for large-scale analysis in a large number of human gastric cancer tissues to verify the involvement of PVT1 in development of gastric cancer. The relationships between $P V T 1$ expression and clinical features were also analyzed.

Results: PVT1 showed higher expression in human gastric cancer tissues than in adjacent non-cancerous tissues and in SGC7901 paclitaxel-resistant cells compared with SGC7901 cells. PVT1 expression was correlated with lymph node invasion of gastric cancer.

Conclusion: $P V T 1$ is a new biomarker for human gastric cancer and may indicate lymph node invasion. Therefore, $P V T 1$ shows potential as a novel therapeutic target for the treatment of gastric cancer and enhancement of paclitaxel sensitivity.

Keywords: microarray analysis, quantitative polymerase chain reaction, lymph node invasion, tumor biomarkers, paclitaxel resistance

\section{Introduction}

Gastric cancer remains a major public health concern as it represents the fourth most common cancer and the second leading cause of cancer-related deaths worldwide. ${ }^{1-3}$ Nevertheless, the incidence and mortality associated with gastric cancer have decreased considerably over the past 50 years in most areas of the world. ${ }^{1}$ Although conventional diagnostic methods such as gastric endoscopy have contributed to this reduction by enabling early diagnosis, many patients are diagnosed with advanced gastric cancer and have a poor prognosis. Thus, more sensitive tumor markers are urgently needed to improve screening, diagnosis, prognostic evaluation, and tumor grading of gastric cancer. Several potential approaches have been proposed to find suitable marker candidates, and the recent discovery of long-non-coding RNAs (lncRNAs) provides a new opportunity to resolve this challenge. LncRNAs are non-protein-coding transcripts longer than 200 nucleotides ${ }^{4}$ and are widely distributed in the genome; indeed, more than $90 \%$ of the human genome is composed of non-coding RNAs. ${ }^{5}$ There is mounting evidence 
that many lncRNAs are dysregulated in various cancers and play important roles in tumorigenesis and invasion. ${ }^{6-8}$ Thus, lncRNAs may serve as useful tumor markers and therapeutic targets for controlling malignant tumors. In the current study, we investigated the expression of plasmacytoma variant translocation 1 (PVT1), a documented oncogenic lncRNA, ${ }^{9}$ in gastric cancer and examined its association with clinical features in patients with gastric cancer to reveal its potential as a biomarker and therapeutic target.

\section{Materials and methods}

Thirty-three tumor tissues and corresponding non-cancerous tissues were obtained at the time of surgery from June 2012 to October 2013 at the First Affiliated Hospital and Union Hospital of Fujian Medical University, Fuzhou, Fujian, People's Republic of China. Following excision, the gastric cancer and non-cancerous tissues were immediately frozen in liquid nitrogen and stored at $-80^{\circ} \mathrm{C}$ until use.

The SGC7901 gastric cancer cell line was obtained from the China Center for Type Culture Collection (Wuhan, People's Republic of China). Paclitaxel-resistant SGC7901 cells were developed in our laboratory and cultured in $450 \mu \mathrm{g} / \mathrm{L}$ paclitaxel. Total mRNA was isolated using TRIzol ${ }^{\circledR}$ Reagent (Life Technologies Corporation, Carlsbad, CA, USA), according to the manufacturer's protocol.

The paclitaxel-resistant cell line was developed by culture of an SGC7901 gastric cancer cell line in vitro with an increasing gradient of concentrations of paclitaxel intermittently over 6 months. The initial concentration of paclitaxel was $25 \mu \mathrm{g} / \mathrm{L}$. Forty-eight hours later, medium containing $25 \mu \mathrm{g} / \mathrm{L}$ paclitaxel was replaced with routine medium until the SGC7901 cells were growing in exponential period, after which the paclitaxel concentration was increased to $50 \mu \mathrm{g} / \mathrm{L}$. Six months later, the final concentration of paclitaxel was $450 \mu \mathrm{g} / \mathrm{L}$, and the resistance in paclitaxel-resistant SGC7901 cells was detected with MTT (3-(4,5-dimethylthiazol-2-yl)-2,5-diphenyltetrazolium bromide) and compared with normal SGC7901 cells. The MTT assay was performed after 48 hours of incubation with paclitaxel (five concentrations in a multiple proportion dilution from 160 to $10 \mu \mathrm{g} / \mathrm{L}$ ), the plates were centrifuged to pellet the cells, the supernatant was removed, and $20 \mu \mathrm{L}$ of $5 \mathrm{mg} / \mathrm{mL}$ MTT was added in $180 \mu \mathrm{L}$ of medium, followed by incubation for 4 hours at $37^{\circ} \mathrm{C}$ in a humid, $5 \% \mathrm{CO}_{2}$ atmosphere. Next, the plates were centrifuged again, the supernatant was removed, and the insoluble formazan crystals were dissolved in dimethyl sulfoxide. Absorbance was read at $490 \mathrm{~nm}$. The half maximal inhibitory concentration $\left(\mathrm{IC}_{50}\right)$ was calculated using the Spearman-Kärber cytopathic effect method. ${ }^{10}$
Before lncRNA microarray analysis, the MTT assay showed $\mathrm{IC}_{50}$ values for SGC7901 and paclitaxel-resistant SGC7901 of $22.16 \pm 0.31 \mu \mathrm{g} / \mathrm{L}$ and $141.31 \pm 0.95 \mu \mathrm{g} / \mathrm{L}$, respectively. The difference was statistically significant $(P<0.001)$. The drug resistance index for paclitaxel-resistant SGC7901 cells was 6.38 .

\section{Microarray analysis}

Tissue samples (two gastric cancer tissues and two corresponding non-cancerous tissues) and SGC7901 cells were used to synthesize double-stranded complementary DNA, which was labeled according to the protocol of the Amplification and Labeling Kit for Microarray (CapitalBio Corporation, Beijing, People's Republic of China). The labeled complementary DNA was hybridized to the Agilent Human LncRNA 4×180 K Expression Microarray (Agilent Technologies Inc., Santa Clara, CA, USA), which contains 37,000 human lncRNAs and 34,000 mRNA probes (CapitalBio Corporation). After hybridization and washing, the processed slides were scanned with a G2565CA microarray scanner (Agilent Technologies). Raw data were extracted as paired files using Feature Extraction software (Agilent Technologies). The raw data were normalized using GeneSpringGX11 software (Agilent Technologies) with default parameters. Differential expression of lncRNAs was screened in genes flagged as "detected" by the software. Differentially expressed genes were identified through the random variance model.

\section{Quantitative polymerase chain reaction}

Total mRNA from 31 gastric cancer tissues and 31 corresponding non-tumor tissues was isolated using TRIzol Reagent according to the manufacturer's protocol. Complementary DNAs from all samples were synthesized from $1.0 \mu \mathrm{g}$ of total RNA using the PrimeScript ${ }^{\circledR} 1$ st strand cDNA synthesis kit (TaKaRa Bio, Shiga, Japan) following the manufacturer's protocol. PVT1 expression levels were quantified using a LightCycler Brilliant SYBR Green quantitative reverse transcriptase polymerase chain reaction (PCR) kit (Roche Applied Science, Indianapolis, IN, USA) following the manufacturer's protocol, with the following primers: forward, TTGGCACATACAGCCATCAT and reverse, GCAGTAAAAGGGGAACACCA. The length of the quantitative PCR product was 102 base pairs. The PVT1 expression level in each sample was normalized to the respective human $\beta$-actin expression level, with the following primers: forward, AGCGAGCATCCCCCAAAGTT; and reverse, GGGCACGAAGGCTCATCATT (product length, 285 base pairs). The specificity of each PCR reaction was confirmed by melting curve analysis. 


\section{Statistical analysis}

The statistical significance of differences between cancerous and adjacent non-cancerous tissues was determined using the Wilcoxon signed-rank test. The correlation between PVTI expression and patient clinicopathological variables was analyzed by the Fisher's exact probability test. A $P$-value $<0.05$ was regarded as being statistically significant. All statistical analyses were performed using Statistical Package for the Social Sciences version 11.0 software for Windows (SPSS Inc., Chicago, IL, USA).

\section{Results}

\section{Microarray analysis}

A total of 252 lncRNAs were upregulated in gastric cancer tissues identified from the microarrays. Twenty-eight of the upregulated lncRNAs had an obvious fold change in the gastric cancer tissues when compared with corresponding non-tumor tissues (Figure 1 and Table 1). In total, $545 \operatorname{lncRNAs}$ were upregulated in paclitaxel-resistant SGC7901 cells when compared with normal SGC7901 cells (data supplied). PVT1 expression levels in the gastric cancer tissues were 5.128 and 5.755 times higher than those in the corresponding nontumor tissues (Table 1). In addition, PVT1 expression was 3.715 times higher in paclitaxel-resistant SGC7901 cells than in normal SGC7901 cells (data supplied). Based on these results and recent discoveries concerning the role of $P V T 1$ in tumorigenesis and resistance to chemotherapy, ${ }^{9,11}$ we studied the expression of $P V T 1$ further in patients with gastric cancer and its correlation with clinical features.

\section{PVTI expression}

PVT1 expression levels in cancerous and adjacent noncancerous tissues from patients with gastric cancer were examined by quantitative reverse transcriptase PCR and

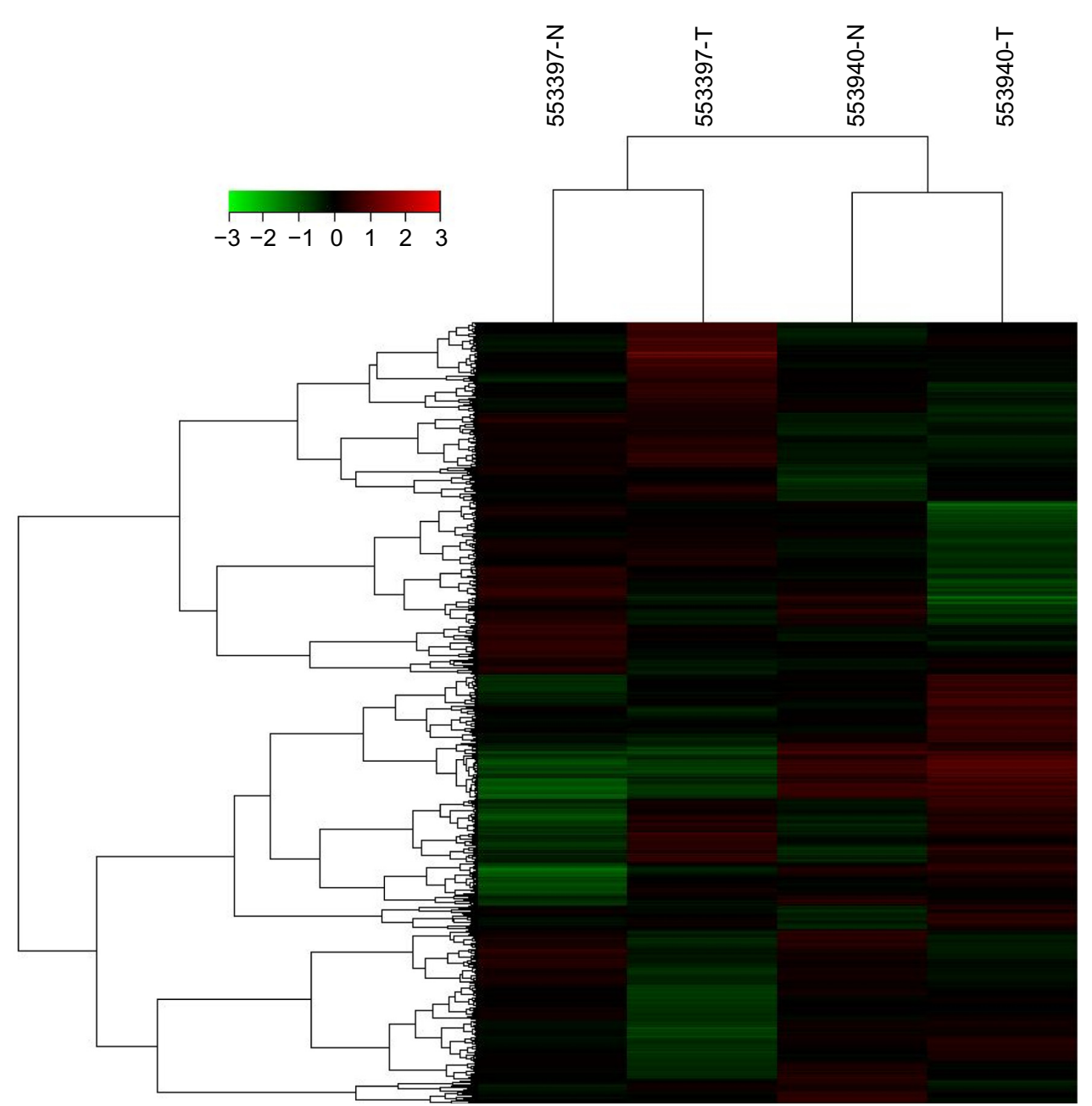

Figure I Cluster diagram of IncRNA expression data in gastric cancer and corresponding non-tumor tissues detected by the Agilent Human LncRNA $4 \times 180 \mathrm{~K}$ Expression Microarray (Agilent Technologies Inc., Santa Clara, CA, USA).

Abbreviation: IncRNA, long-non-coding RNA. 
Table I Twenty-eight candidate IncRNAs expressed with fold changes in two gastric cancer tissues, compared with corresponding non-tumor tissues (only the most obvious data are shown for the IncRNAs with different transcripts)

\begin{tabular}{|c|c|c|c|c|}
\hline Fold change of TI vs NI & Fold change of T2 vs N2 & Gene biotype & Ensemble gene ID & HGNC symbol \\
\hline 27.20002 & 104.10264 & Antisense & ENSG00000238I33 & MLK7-ASI \\
\hline $16.4 \mid 4074$ & 4.819842 & LncRNA & ENSG00000258294 & RPII-3I4D7.I \\
\hline 12.021113 & 5.851729 & LncRNA & ENSG00000236039 & AC019117.2 \\
\hline 10.219363 & 5.664894 & LncRNA & ENSG0000024II 63 & RPII-398A8.3 \\
\hline 8.035696 & 3.0575392 & LncRNA & ENSG0000022204I & LINC00I52 \\
\hline 6.8010974 & 2.6033688 & LncRNA & ENSG00000226598 & AC0I7060.I \\
\hline 5.912395 & 4.000447 & Antisense & ENSG00000224078 & SNHGI 4 \\
\hline 5.128479 & 5.754559 & LncRNA & ENSG00000249859 & PVTI \\
\hline 4.606247 & 4.3609843 & LncRNA & ENSG00000167117 & LINC00483 \\
\hline 3.909583 & 2.458897 & LncRNA & ENSG00000244306 & CTD-23I4B22.3 \\
\hline 3.8943083 & 4.077277 & LncRNA & ENSG00000224397 & RPII-290F20.3 \\
\hline 3.698091 & 5.106683 & Antisense & ENSG00000243766 & HOTTIP \\
\hline 3.5476947 & 4.3146453 & Antisense & ENSG00000254560 & RPII-ILI2.3 \\
\hline 3.3448012 & 4.3363175 & Antisense & ENSG0000025। I 79 & RPII-893F2.9 \\
\hline 3.2606792 & 2.2400966 & Polymorphic_pseudogene & ENSG00000244682 & FCGR2C \\
\hline 3.0598006 & 3.2264988 & LncRNA & ENSG0000023006I & AP00I065.2 \\
\hline 3.0492082 & 3.112142 & LncRNA & ENSG00000233930 & RPI3-25N22.I \\
\hline 2.9770749 & 4.8328776 & LncRNA & ENSG00000227888 & FAM66A \\
\hline 2.8799016 & 2.383093 & Processed transcript & ENSG00000233283 & RPII-357HI4.20 \\
\hline $2.878 \mid 404$ & 2.6564493 & Processed transcript & ENSG00000250610 & RPII-738E22.2 \\
\hline 2.7795174 & 2.031886 & LncRNA & ENSG00000230944 & AC026202.5 \\
\hline 2.7087636 & 25.26169 & LncRNA & ENSG0000020424I & RPII-7|3PI7.3 \\
\hline 2.5824153 & 2.942296 & LncRNA & ENSG00000I363I5 & RPII-84CI0.2 \\
\hline 2.453376 & 2.046279 & LncRNA & ENSG000002I 4548 & MEG3 \\
\hline 2.4336877 & 3.0326896 & LncRNA & ENSG00000248227 & RPI I-83C7.I \\
\hline 2.2753818 & 2.2845905 & LncRNA & ENSG00000256969 & RPII-320N7.2 \\
\hline 2.1950364 & 4.0923414 & LncRNA & ENSG00000245975 & RPII-30K9.6 \\
\hline 2.125026 & 2.8170245 & LncRNA & ENSG00000235572 & RPII-555H7.2 \\
\hline
\end{tabular}

Abbreviations: HGNC, Human Genome Nomenclature Committee; IncRNAs, long-noncoding RNAs.

normalized by $\beta$-actin expression levels. The Wilcoxon signed-rank test showed that PVT1 expression was significantly higher in cancerous tissues than in adjacent noncancerous tissues $(P=0.0414)$.

\section{PVTI expression and clinical features}

In Figure 2 and Table 2, the human gastric cancer tissues are classified according to $P V T 1$ expression level into a high $P V T 1$ expression group ( $P V T 1$ in cancer tissue/ $P V T 1$ in non-cancerous tissue $>1.0$ ) and a low $P V T 1$ expression group ( $P V T 1$ in cancer tissue/ $P V T 1$ in non-cancerous tissue $<1.0)$. The association of $P V T 1$ expression with the clinicopathological features of patients with intestinal-type gastric cancer is summarized in Table 2. A significant association was found between PVT1 expression and lymph node metastasis $(P=0.022)$. No correlation was found between $P V T 1$ expression and patient age, sex, tumor metastasis, or stage of gastric cancer.

\section{Discussion}

A set of lncRNAs showed differential expression between human gastric cancer and non-cancerous tissue. Thus, these
lncRNAs may play important roles in the carcinogenesis and invasiveness of human gastric cancer and are potential candidates for new biomarkers and therapeutic targets. ${ }^{6-9}$

$P V T 1$, one such oncogenic lncRNA, is a large $(>300 \mathrm{~kb})$ locus located $57 \mathrm{~kb}$ downstream of $M Y C$ on human chromosome $8 \mathrm{q} 24 .{ }^{12-15}$ Although no protein product or regulatory RNA has yet been identified among PVT1 transcripts, the importance of this locus has caught the attention of many researchers, as it is a site of both tumorigenic translocations and retroviral insertions. Translocation breakpoints within either the MYC or PVT1 locus are responsible for the characteristic lesions associated with Burkitt lymphoma and mouse plasmacytomas. ${ }^{13-18}$

Coamplification of human MYC and PVT1 has been shown to correlate with rapid progression of breast cancer as well as poor clinical survival in postmenopausal women and in patients with HER2-positive breast cancer. ${ }^{19}$ Recent studies have discovered a cluster of miRNAs within the PVT1 genomic DNA region, including miR1204, miR1205, miR1206, miR1207, and miR1208. ${ }^{20,21}$ The regulatory functions of these miRNAs within MYC-PVT1 




Figure 2 Ratio of relative PVTI expression in cancerous tissues versus adjacent noncancerous tissues from 31 patients with gastric cancer, examined by quantitative reverse transcriptase polymerase chain reaction. $\mathrm{N}$ indicates the number of patients with lymph node metastasis.

locus are complicated, only the function of miR1204 has been partially proved. MiR1204 plays an antiproliferative role, inducing cell death or cell cycle arrest, as a functional target of p53 at the PVT1 locus. MiR1204 expression can also increase p53 levels, suggesting there is a positive feedback between miR-1204 and p53. ${ }^{22}$ So far, at least

Table 2 Association between PVTI expression and clinicopathological factors in human gastric cancers

\begin{tabular}{llll}
\hline & $\begin{array}{l}\text { High PVTI } \\
\text { expression }\end{array}$ & $\begin{array}{l}\text { Low PVTI } \\
\text { expression }\end{array}$ & P-value $^{\text {a }}$ \\
\hline $\begin{array}{l}\text { Age } \\
\text { Sex }\end{array}$ & $60.7895 \pm 7.044$ I & $64.7500 \pm 9.0566$ & 0.1827 \\
$\quad$ Male & II & 10 & 0.1285 \\
$\quad$ Female & 8 & 2 & \\
T & & & 1.0000 \\
TI, T2 & 4 & 3 & \\
T3, T4 & 15 & 9 & 0.0220 \\
N & & & \\
N0 & 4 & 8 & 0.2645 \\
NI, N2, N3 & 15 & 4 & \\
M & & & \\
M0 & 16 & 12 & \\
MI & 3 & 0 & \\
Stage & & 8 & \\
I, 2 & 5 & 4 & \\
3, 4 & 14 & &
\end{tabular}

Note: aFisher's exact probability test. one PVT1 transcript microRNA product, miR1204, has been shown to be overexpressed and is thus defined as oncogenic. ${ }^{9,22}$ These additional non-coding RNA transcripts, exonic PVT1 sequences, and other miRs are now thought to be either transcriptional byproducts of miR1204 regulation or additional functional targets, but no further evidence is provided. So, except for two aspects of the mechanism of PVT1 in the prognosis of cancer (including a positive feedback regulation with p53 and coamplification with $M Y C$ ), more studies need to be directed to the complex networks between PVT1, p53, MYC, and other genes.

In this study, we examined the expression of lncRNAs with $77 \times 10^{3}$ probes from the Agilent Human $4 \times 180 \mathrm{~K}$ Microarray to identify specific lncRNA markers for human gastric cancers at the gene level. These probes correspond to 37,000 lncRNAs and 34,000 mRNAs. We also compared IncRNA expression between an SGC7901 human gastric cancer cell line and a paclitaxel-resistant SGC7901 cell line. The differential expression observed may reveal the mechanism of and biomarkers for paclitaxel resistance in human gastric cancer. Based on the microarray results and in light of recent reports, ${ }^{11,13-19}$ we ranked PVT1 as the lncRNA with the highest potential as a biomarker and a therapeutic target for gastric cancer. 
In order to compensate for the deficiency of clinical cases in the microarray analysis and to develop a convenient and economical method for large-scale clinical studies, we used the quantitative PCR technique to confirm the relevance of $P V T 1$ as a biomarker for gastric cancer. Our results clearly show that PVT1 expression is significantly enhanced in gastric cancer tissues when compared with non-cancerous tissues of the stomach. A high level of PVT1 expression was also associated with lymph node metastasis in patients with gastric cancer. No correlation was found between PVT1 expression and age, sex, tumor, metastasis, or stage of gastric cancer.

In conclusion, we demonstrated that PVT1 is highly expressed in human gastric cancer. Enhanced expression of PVT1 was associated with invasion to the lymph nodes and with resistance to paclitaxel. Therefore, these results indicate that PVT1 is a potential candidate as a biomarker for gastric cancer. Screening for PVT1 expression could potentially improve early diagnosis, therapy, and drug resistance, and thus improve quality of life for patients with gastric cancer.

\section{Acknowledgment}

The study was supported by the China National Science Foundation (81300321), the Fujian Provincial Natural Science Fund (2013J01369), and the Fujian Provincial Natural Science Fund (2014J01419).

\section{Disclosure}

The authors report no conflicts of interest in this work.

\section{References}

1. Parkin DM, Bray FI, Devesa SS. Cancer burden in the year 2000. The global picture. Eur J Cancer. 2001;37 Supp1 8:S4-S66.

2. Parkin DM. International variation. Oncogene. 2004;23(38): 6329-6340.

3. Stewart BW, Kleihues P. World Cancer Report. Lyon, France: IARC Press; 2003.

4. Perkel JM. Visiting "noncodarnia”. Biotechniques. 2013;54(6):301, 303-304

5. ENCODE Project Consortium; Birney E, Stamatoyannopoulos JA, Dutta A, et al. Identification and analysis of functional elements in 1\% of the human genome by the ENCODE pilot project. Nature. 2007; 447(7146):799-816.

OncoTargets and Therapy

\section{Publish your work in this journal}

OncoTargets and Therapy is an international, peer-reviewed, open access journal focusing on the pathological basis of all cancers, potential targets for therapy and treatment protocols employed to improve the management of cancer patients. The journal also focuses on the impact of management programs and new therapeutic agents and protocols on
6. Gupta RA, Shah N, Wang KC, et al. Long non-coding RNA HOTAIR reprograms chromatin state to promote cancer metastasis. Nature. 2010;464(7291):1071-1076.

7. Zhang L, Yang F, Yuan JH, et al. Epigenetic activation of the MiR-200 family contributes to H19-mediated metastasis suppression in hepatocellular carcinoma. Carcinogenesis. 2013;34(3):577-586.

8. Lai MC, Yang Z, Zhou L, et al. Long non-coding RNA MALAT-1 overexpression predicts tumor recurrence of hepatocellular carcinoma after liver transplantation. Med Oncol. 2012;29(3):1810-1816.

9. Beck-Engeser GB, Lum AM, Huppi K, Caplen NJ, Wang BB, Wabl M. Pvt1-encoded microRNAs in oncogenesis. Retrovirology. 2008;5:4.

10. Hamilton MA, Russo RC, Thurston RV. Trimmed Spearman-Kärber CPE method for estimating median lethal concentrations in toxicity bioassays. Environ Sci Technol. 1977;13:714-719.

11. You L, Chang D, Du HZ, Zhao YP. Genome-wide screen identifies PVT1 as a regulator of gemcitabine sensitivity in human pancreatic cancer cells. Biochem Biophys Res Commun. 2011;407(1):1-6.

12. Shtivelman E, Henglein B, Groitl P, Lipp M, Bishop JM. Identification of a human transcription unit affected by the variant chromosomal translocations 2;8 and 8;22 of Burkitt lymphoma. Proc Natl Acad Sci US A. 1989;86(9):3257-3260.

13. Huppi K, Siwarski D, Skurla R, et al. Pvt-1 transcripts are found in normal tissues and are altered by reciprocal $(6 ; 15)$ translocations in mouse plasmacytomas. Proc Natl Acad Sci U SA. 1990;87(18):6964-6968.

14. Shtivelman E, Bishop JM. Effects of translocations on transcription from PVT. Mol Cell Biol. 1990;10(4):1835-1839.

15. Shtivelman E, Bishop JM. The PVT gene frequently amplifies with MYC in tumor cells. Mol Cell Biol. 1989;9(3):1148-1154.

16. Pleasance ED, Stephens PJ, O'Meara S, et al. A small-cell lung cancer genome with complex signatures of tobacco exposure. Nature. 2010; 463(7278):184-190.

17. Pomerantz MM, Beckwith CA, Regan MM, et al. Evaluation of the $8 \mathrm{q} 24$ prostate cancer risk locus and MYC expression. Cancer Res. 2009;69(13):5568-5574.

18. Enciso-Mora V, Broderick P, Ma Y, et al. A genome-wide association study of Hodgkin's lymphoma identifies new susceptibility loci at 2p16.1 (REL), 8q24.21 and 10p14 (GATA3). Nat Genet. 2010;42(12):1126-1130.

19. Borg A, Baldetorp B, Fernö M, Olsson H, Sigurdsson H. c-Myc amplification is an independent prognostic factor in postmenopausal breast cancer. Int J Cancer. 1992;51(5):687-691.

20. Huppi K, Volfovsky N, Runfola T, et al. The identification of microRNAs in a genomically unstable region of human chromosome 8q24. Mol Cancer Res. 2008;6(2):212-221.

21. Bisio A, De Sanctis V, Del Vescovo V, et al. Identification of new p53 target microRNAs by bioinformatics and functional analysis. $B M C$ Cancer. 2013;13:552.

22. Barsotti AM, Beckerman R, Laptenko O, Huppi K, Caplen NJ, Prives C. p53-dependent induction of PVT1 and miR-1204. J Biol Chem. 2012;287(4):2509-2519. patient perspectives such as quality of life, adherence and satisfaction. The manuscript management system is completely online and includes a very quick and fair peer-review system, which is all easy to use. Visit http://www.dovepress.com/testimonials.php to read real quotes from published authors. 